\title{
Bilateral Leukemic Pulmonary Infiltrate: A Rare Manifestation of Acute Myeloid Leukemia
}

\author{
Imran Umera, b, Farzauq Burki ${ }^{\mathrm{a}}$, Yasir Ahmed ${ }^{\mathrm{a}}$
}

\begin{abstract}
Acute myeloid leukemia (AML) often presents with nonspecific symptoms of fatigue, anemia or infection. Pulmonary involvement is common in AML during the course of the disease and is usually caused by infection, hemorrhage, leukemic pulmonary infiltrates and leukostasis. Symptomatic leukemic pulmonary infiltrates as a first manifestation of AML is very uncommon but potentially life threatening if not diagnosed and treated in a timely manner. Here we present a rare case of AML with symptomatic bilateral leukemic infiltrates as a first manifestation with peripheral blasts $77 \%$. Common infectious etiologies were ruled out. The pulmonary infiltrates dramatically improved with standard induction chemotherapy. Even though leukemic pulmonary infiltrates in AML is a diagnosis of exclusion but must be considered in the differential diagnosis when a patient present with pulmonary symptoms with blast cell $>$ $40 \%$ and suspected acute leukemia.
\end{abstract}

Keywords: Acute leukemia; Acute myeloid leukemia; Leukemic pulmonary infiltrate; Lung infiltrate

\section{Introduction}

Diffuse pulmonary infiltration is frequently found in patients with acute myeloid leukemia (AML) during the course of their underlying disease, though clinical presentation is variable. In some patients death may ensure within a few days after respiratory symptoms become prominent. The initial presentation of AML as symptomatic bilateral leukemic pulmonary infiltrates is relatively infrequent and poses a diagnostic dilemma. Here we present a rare case of AML with

\footnotetext{
Manuscript accepted for publication January 13, 2014

${ }^{a}$ Department of Internal Medicine, Texas Tech University of Health Science Center, Odessa, Texas, USA

${ }^{\mathrm{b}}$ Corresponding author: Imran Umer, Department of Internal Medicine, TTUHSC, 701 West 5th Street, Odessa, Texas 79763, USA.

Email: imran.umer@ttuhsc.edu

doi: http://dx.doi.org/10.14740/jmc1651w
}

bilateral leukemic pulmonary infiltrates that responded to standard chemotherapy dramatically.

\section{Case Report}

A 59-year-old healthy female presented with low grade fever, night sweats, cough and progressive dyspnea for 2 weeks. Review of systems was significant for generalized weakness and fatigue. Physical examination revealed respiratory rate: 22 /minute, heart rate: 110 beats/minute, blood pressure: 130/70 mmHg, temperature: $37.8^{\circ} \mathrm{C}$, oxygen saturation $96 \%$ on 2 liters of $\mathrm{O} 2$ via nasal cannula. Bilateral crackles in lungs along with sterna tenderness noted, rest of systemic examination was unremarkable. Initial labs showed hemoglobin $8.15 \mathrm{~g} / \mathrm{dL}$, platelet count $86,000 / \mu \mathrm{L}$, white blood count of $105,000 / \mu \mathrm{L}$ with $77 \%$ blast cells on peripheral smears, serum lactate dehydrogenase level was 3,788 U/L; aspartate aminotransferase was $64 \mathrm{U} / \mathrm{L}$ and renal function was normal. Chest X-ray showed bibasilar and right lobe infiltrates with minimal bilateral pleural effusion (Fig. 1a), computed tomography (CT) confirmed same finding. The patient was started on empiric intravenous vancomycin and piperacillin-tazobactam, but later on pulmonary infiltrates slightly worsen and oxygen requirement increased to $45 \%$, so levofloxacin was also added. Bone marrow biopsy revealed $60 \%$ immature mononuclear blast cells mainly monocytes (Fig. 2 ) and immune flow cytometry confirmed AML-M-1 type. Infectious work up included HIV screen, tuberculin skin test (TST), blood cultures, fungal serology, mycoplasma serology, serum cryptococcal antigen, urinary legionella and histoplasma antigen, bronchoalveolar lavage (BAL) fluids for bacterial, fungal and AFB cultures remained negative; hence all antibiotics were discontinued on day8. She underwent induction chemotherapy with cytarabine arabinoside and idarubicin. Pulmonary infiltrate improved dramatically and repeated CXR at hospital day 22 showed complete resolutions of infiltrates and pleural effusion (Fig. 1b). She developed chemotherapy-related transient neutropenia which responded to granulocyte-colony stimulating factor. She went home on day 25 for outpatient consolidative therapy and at 6 months follow up visit she remained in remission. 


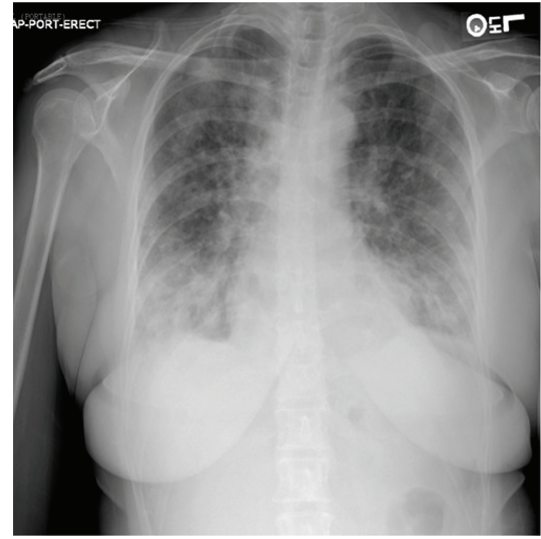

$\mathrm{a}$

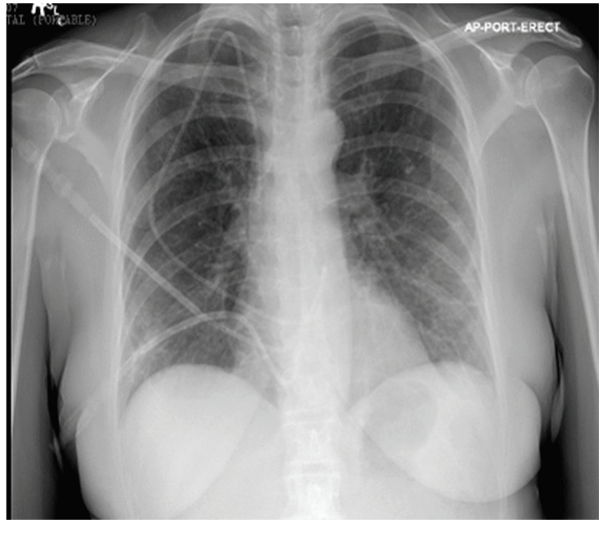

b

Figure 1. (a). Anterioposterior chest radiograph showing bibasilar pulmonary infiltrates with minimal bilateral plural effusion, with right upper lobe infiltrates; (b). Anterioposterior chest radiograph after induction chemotherapy showing complete resolution of bilateral pulmonary infiltrates and plural effusion.

\section{Discussion}

Pulmonary complications occur during the course of disease in nearly $80 \%$ of patients with leukemia, and are the major cause of death. Leukemic pulmonary infiltration is not unusual at late stages of both acute and chronic leukemia [1]. However, the initial presentation of acute leukemia as symptomatic pulmonary infiltrates is uncommon [2]. An autopsy study of the lung showed less than 10 percent of the patients found to have leukemic involvement of the lung had respiratory symptoms before death [3].

Lung infiltration can be due to leukemic interstitial infil-

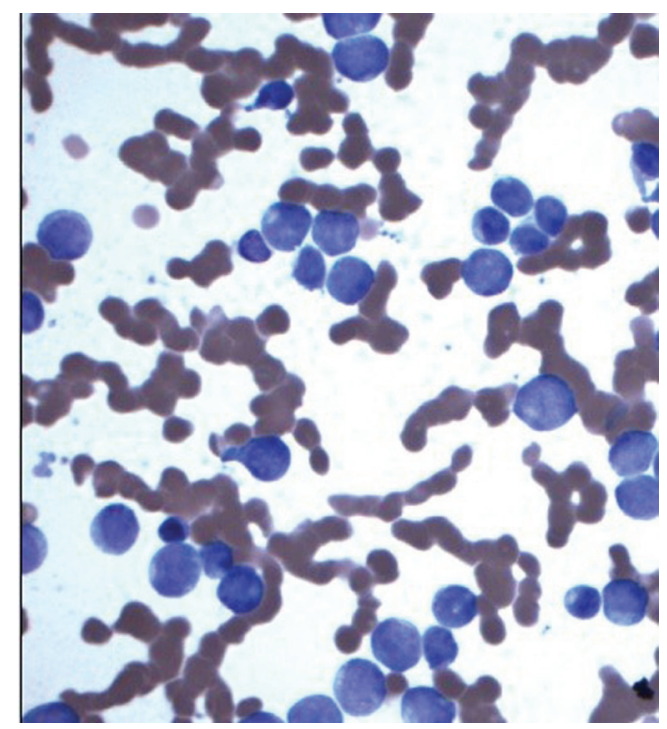

Figure 2. Bone marrow aspirate: arrow showing blast cells, mainly monocytes. tration or intravascular leukostasis. Leukemic invasion of the lungs must be distinguished from aggregation of leukemic blast cells within the pulmonary vasculature which is commonly present in patients with very high white blood cell counts $>2,00,000 / \mu \mathrm{L}$. True leukemic pulmonary infiltrates are typically interstitial in distribution; the blast cells are characteristically found around smaller bronchi and blood vessels and may seem to form nodules [3]. Leukemic pulmonary infiltrate usually present with symptoms of fever, dyspnea, cough and hemoptysis and radiographic infiltrates, clinically all suggestive of pneumonia. However cultures will remain negative and infiltrates resolve with chemotherapy [4]. Sometime leukemic pulmonary infiltrate may progress to acute respiratory distress syndrome which could be life threatening [5].

Differential diagnosis of pulmonary infiltrates includes wide spectrum of pulmonary infections, alveolar hemorrhage, pulmonary edema, hypersensitivity pneumonitis, leukostasis [6]. Leukemic pulmonary infiltration should be suspected when blast cells are more than $40 \%$ in peripheral blood [2] and there is no clinical evidence of pulmonary infection. Surgical lung biopsy may help in diagnosis [7]. Rossi et al reported that the presence of leukemic cells in bronchoalveolar lavage may help establishing the diagnosis, but these cells are not commonly seen unless the patient develops pulmonary hemorrhage [8]. There are no pathognomonic radiological findings for pulmonary leukemic infiltration. CXR may be normal or varies from diffuse interstitial, alveolar, and reticular nodular or mixed pattern [9]. Highresolution CT findings of leukemic infiltrates are nonspecific [10]. If diagnosed early, most patients show good clinical outcome with standard chemotherapy.

In common with the other reported cases, our patient had peripheral blast count $>40 \%$ at the time the diagnosis of 
leukemic pulmonary infiltrates with negative all infectious work up and the infiltrates resolved dramatically with chemotherapy.

\section{Conclusion}

Leukemic pulmonary infiltrates should be considered in patient presenting with pulmonary infiltrates in setting of acute leukemia with peripheral blast cells count $>40 \%$ in blood. An aggressive diagnostic approach should be employed promptly in such patient to rule out infectious causes before starting chemotherapy to achieve good clinical outcome.

\section{Conflict of Interest}

Nothing to disclose.

\section{Grant}

None.

\section{References}

1. Rosenow EC, 3rd, Wilson WR, Cockerill FR, 3rd. Pulmonary disease in the immunocompromised host. 1 . Mayo Clin Proc. 1985;60(7):473-487.

2. Azoulay E, Fieux F, Moreau D, Thiery G, Rousselot P, Parrot A, Le Gall JR, et al. Acute monocytic leukemia presenting as acute respiratory failure. Am J Respir Crit
Care Med. 2003;167(10):1329-1333.

3. Kovalski R, Hansen-Flaschen J, Lodato RF, Pietra GG. Localized leukemic pulmonary infiltrates. Diagnosis by bronchoscopy and resolution with therapy. Chest. 1990;97(3):674-678.

4. Hildebrand FL, Jr., Rosenow EC, 3rd, Habermann TM, Tazelaar HD. Pulmonary complications of leukemia. Chest. 1990;98(5):1233-1239.

5. Wu YK, Huang YC, Huang SF, Huang CC, Tsai YH. Acute respiratory distress syndrome caused by leukemic infiltration of the lung. J Formos Med Assoc. 2008;107(5):419-423.

6. Bhatia M, Coppage L. Dyspnea, nonproductive cough, and blasts on the peripheral smear. Chest. 1995;107(1):269-270.

7. White DA, Wong PW, Downey R. The utility of open lung biopsy in patients with hematologic malignancies. Am J Respir Crit Care Med. 2000;161(3 Pt 1):723-729.

8. Rossi GA, Balbi B, Risso M, Repetto M, Ravazzoni C. Acute myelomonocytic leukemia. Demonstration of pulmonary involvement by bronchoalveolar lavage. Chest. 1985;87(2):259-260.

9. Lee WA, Hruban RH, Kuhlman JE, Fishman EK, Wheeler PS, Hutchins GM. High resolution computed tomography of inflation-fixed lungs: pathologic-radiologic correlation of pulmonary lesions in patients with leukemia, lymphoma, or other hematopoietic proliferative disorders. Clin Imaging. 1992;16(1):15-24.

10. Heyneman LE, Johkoh T, Ward S, Honda O, Yoshida S, Muller NL. Pulmonary leukemic infiltrates: high-resolution CT findings in 10 patients. AJR Am J Roentgenol. 2000;174(2):517-521. 